\title{
EMBEDDING THEOREMS FOR GROUPS
}

\author{
by C. G. CHEHATA \\ (Received 5th December, 1962)
}

\section{Introduction}

By a partial endomorphism of a group $G$ we mean a homomorphic mapping $\mu$ of a subgroup $A$ of $G$ onto a subgroup $B$ of $G$. If $\mu$ is defined on the whole of $G$ then it is called a total endomorphism. We call a partial endomorphism totally extendable (or extendable) if there exists a supergroup $G^{*} \supseteq G$ with a total endomorphism $\mu^{*}$ which extends $\mu$ in the sense that $\operatorname{g} \mu^{*}=g \mu$, whenever the right-hand side is defined (3).

In a previous paper (2), we derived necessary and sufficient conditions for a well-ordered set of partial endomorphisms $\mu(\alpha)$ of a group $G$ to be extendable to a set of total endomorphisms $\mu^{*}(\alpha)$ of a supergroup $G^{*}$ such that each $\mu^{*}(\alpha)$ acts as an isomorphism on $G^{*}\left[\mu^{*}(\alpha)\right]^{n(\alpha)}$, where $n(\alpha)$ is a given positive integer. These conditions are in fact a generalisation of the conditions in case of a single extension (1).

In this work sufficient conditions are derived for the required extension, with the same condition imposed on $\mu^{*}(\alpha)$, to be established in case $\mu(\alpha)$ are partial endomorphisms of certain types of subgroups. In particular sufficient conditions for the extension of partial endomorphisms of $E$-subgroups are given; where the subgroup $H$ of the group $G$ is called an E-subgroup if every normal subgroup of $H$ is the intersection with $H$ of a normal subgroup of $G$. This is equivalent to the fact that if $N$ is a normal subgroup of $H$ then $N^{G} \cap H=N$, where $N^{G}$ is the normal closure of $N$ in $G$.

We conclude by deriving necessary and sufficient conditions for a wellordered set of partial endomorphisms of $G$ to be all extendable to one and the same total endomorphism $\theta^{*}$ of a supergroup $G^{*}$ such that $\theta^{*}$ is an isomorphism on $G^{*}\left(\theta^{*}\right)^{m}$ for some positive integer $m$.

\section{Extension in a special case}

We shall assume that $G$ is a given group and $\mu(\alpha)$, where $\alpha$ ranges over a well-ordered set $\Sigma$, is a partial endomorphism of $G$ mapping the subgroup $A(\alpha) \subseteq G$ onto the subgroup $B(\alpha) \subseteq G$. In (2) it was proved that the necessary and sufficient conditions for the existence of $G^{*} \supseteq G$ with total endomorphisms $\mu^{*}(\alpha)$ which extend $\mu(\alpha)$ such that for every $\alpha, \mu^{*}(\alpha)$ acts as an isomorphism on $G^{*}\left[\mu^{*}(\alpha)\right]^{n(\alpha)}$, where $n(\alpha)$ is a positive integer are that if $\Omega$ is the semigroup freely generated by the $\mu(\alpha)$, then for every $\omega \in \Omega$ there exists a normal subgroup 
$L(\omega)$ of $G$ such that

$$
\begin{aligned}
& L(\omega) \subseteq L\left(\omega \omega_{1}\right) \text { for all } \omega, \omega_{1} \in \Omega, \\
& L\left[\{\mu(\alpha)\}^{n(\alpha)}\right]=L\left[\{\mu(\alpha)\}^{n(x)+i}\right],
\end{aligned}
$$

for any $\alpha \in \Sigma$ and any positive in teger $i$,

$$
\begin{aligned}
& L[\mu(\alpha)] \cap A(\alpha) \text { is the kernel of } \mu(\alpha), \\
& {[L\{\mu(\alpha) \omega\} \cap A(\alpha)] \mu(\alpha)=L(\omega) \cap B(\alpha),}
\end{aligned}
$$

for every $\alpha \in \Sigma$ and $\omega \in \Omega$.

Theorem 1. With the presious notation, it is sufficient for the required extension to be established that if, for every $\alpha \in \Sigma, K[\mu(\alpha)]$ is the kernel of $\mu(\alpha)$ then

for every $\alpha, \beta \in \Sigma$.

$$
\begin{aligned}
& K^{G}[\mu(\alpha)] \cap A(\alpha)=K[\mu(\alpha)], \\
& K^{G}[\mu(\alpha)] \cap B(\beta)=\{e\} \ldots \ldots
\end{aligned}
$$

Proof. For every $\omega \in \Omega$, put

$$
L[\mu(\alpha) \omega]=L[\mu(\alpha)]=K^{G}[\mu(\alpha)] .
$$

If $\omega=\mu(\alpha) \omega^{\prime}$ and $\omega_{1}$ are any words in $\Omega$ then

$$
L(\omega)=L\left(\omega \omega_{1}\right)=K^{G}[\mu(\alpha)]
$$

which simultaneously proves (2.1) and (2.2). Also

$$
L[\mu(\alpha)] \cap A(\alpha)=K^{G}[\mu(\alpha)] \cap A(\alpha)=K[\mu(\alpha)]
$$

is the kernel of $\mu(\alpha)$, which proves $(2.3)$.

To prove (2.4) we note that if $\omega=\mu(\gamma) \omega^{\prime}$ is any word in $\Omega$ then

$$
\begin{aligned}
& {[L\{\mu(\alpha) \omega\} \cap A(\alpha)] \mu(\alpha)=[L\{\mu(\alpha)\} \cap A(\alpha)] \mu(\alpha)} \\
& =K[\mu(\alpha)] \mu(\alpha)=e, \\
& \text { and } \quad L(\omega) \cap B(\alpha)=L\left[\mu(\gamma) \omega^{\prime}\right] \cap B(\alpha) \\
& =K^{G}[\mu(\gamma)] \cap B(\alpha) \\
& =e \text {, by (2.6). }
\end{aligned}
$$

This completes the proof of Theorem 1.

\section{Extension in case of E-subgroups}

Theorem 2. Let $A(\alpha)$ be E-subgroups of $G$. If we define $K[\mu(\alpha)]=e \mu^{-1}(\alpha)$ and inductively

$$
K[\mu(\alpha) \omega]=K^{G}(\omega) \mu^{-1}(\alpha)
$$

i.e. the greatest subgroup of $A(\alpha)$ mapped into $K^{G}(\omega)$ by $\mu(\alpha)$, then for the required extension to be established it is sufficient that

$$
K[\mu(\alpha)]^{m} \text { are normal in } G \text {, i.e. }
$$

$K[\mu(\alpha)]^{m+1}=K[\mu(\alpha)]^{m} \mu^{-1}(\alpha) ;$ for $m=1,2, \ldots$, whenever $x[\mu(\alpha)]^{n(\alpha)+1}$ is defined and is equal to $e$ then

$$
x[\mu(\alpha)]^{n(\alpha)}=e .
$$


Proof. We can as in (3) prove that

for any $\omega, \omega^{\prime} \in \Omega$.

$$
K(\omega) \subseteq K\left(\omega \omega^{\prime}\right)
$$

Now we prove that

$$
K[\mu(\alpha)]^{n(\alpha)+1}=K[\mu(\alpha)]^{n(\alpha)} .
$$

Let

$$
\begin{gathered}
x \in K[\mu(\alpha)]^{n(\alpha)+1}=K[\mu(\alpha)]^{n(\alpha)} \mu^{-1}(\alpha), \\
x \mu(\alpha) \in K[\mu(\alpha)]^{n(x)} .
\end{gathered}
$$

thus

Repeating we arrive at

and hence

This implies by (3.3) that

$$
x[\mu(\alpha)]^{n(\alpha)} \in K[\mu(\alpha)],
$$$$
x[\mu(\alpha)]^{n(\alpha)+1}=e .
$$

$$
x[\mu(\alpha)]^{n(x)}=e,
$$

which in turn gives

$$
\dot{x} \in K[\mu(\alpha)]^{n(\alpha)} \text {. }
$$

Thus

$$
K[\mu(\alpha)]^{n(\alpha)+1} \subseteq K[\mu(\alpha)]^{n(\alpha)} ;
$$

but

$$
K[\mu(\alpha)]^{n(\alpha)} \subseteq K[\mu(\alpha)]^{n(x)+1} \text {, from (3.4). }
$$

These two together prove (3.5).

From (3.2) and (3.5) we get

More generally we have

$$
\begin{aligned}
K[\mu(\alpha)]^{n(\alpha)+2} & =K[\mu(\alpha)]^{n(\alpha)+1} \mu^{-1}(\alpha) \\
& =K[\mu(\alpha)]^{n(\alpha)} \mu^{-1}(\alpha) \\
& =K[\mu(\alpha)]^{n(\alpha)+1} \\
& =K[\mu(\alpha)]^{n(\alpha)} .
\end{aligned}
$$

$$
K[\mu(\alpha)]^{n(\alpha)+i}=K[\mu(\alpha)]^{n(\alpha)} \text { for any integer } i>0 .
$$

Now put

$$
L(\omega)=K^{G}(\omega)
$$

Thus from (3.4) and (3.6) we get

$$
L(\omega) \subseteq L\left(\omega \omega^{\prime}\right)
$$

for any $\omega, \omega^{\prime} \in \Omega$; and

$$
L[\mu(\alpha)]^{n(\alpha)+i}=L[\mu(\alpha)]^{n(\alpha)},
$$

for any $\alpha \in \Sigma$ and any integer $i>0$. This proves (2.1) and (2.2). From (3.7) and the fact that $A(\alpha)$ are $E$-subgroups it follows immediately that

$$
L[\mu(\alpha)] \cap A(\alpha)=K[\mu(\alpha)] \text { is the kernel of } \mu(\alpha) \text {, which proves (2.3). }
$$

The proof that (2.4) also holds is the same as in (3). This completes the proof of Theorem 2.

Special case. If the group $G$ is abelian then every subgroup of $G$ is an $E$-subgroup and condition (3.2) holds automatically. Thus we have the following result. 
Corollary. If $G$ is abelian then it is sufficient for the required extension to be established that (3.3) holds.

\section{Extending all $\mu(\alpha)$ to a single endomorphism}

Theorem 3. For all $\mu(\alpha), \alpha \in \Sigma$ to be extendable to one and the same total endomorphism $\theta^{*}$ of a group $G^{*} \supseteq G$ such that $\theta^{*}$ is an isomorphism on $G^{*}\left(\theta^{*}\right)^{m}$, for some positive integer $m$, it is necessary and sufficient that if we define $\theta$ to map any word $w\left(a_{\tau}\right) \in\{A(\alpha)\}$ onto $w\left(a_{\tau} \mu(\tau)\right) \in\{B(\alpha)\}$ where $a_{\tau} \in A(\tau)$, $\tau$ ranges over some finite set $I \subset \Sigma, \alpha$ ranges over $\Sigma$ then

$\theta$ is a one-valued mapping of $\{A(\alpha)\}$ onto $\{B(\alpha)\}$ which is a homomorphism, (4.1) there exists in $G$ a sequence of normal subgroups

$$
L_{1} \subseteq L_{2} \subseteq \ldots \subseteq L_{m}=L_{m+1}=\ldots
$$

such that

for $j=1,2, \ldots, m$.

$$
\begin{aligned}
& L_{1} \cap\{A(\alpha)\} \text { is the kernel of } \theta, \\
& {\left[L_{j+1} \cap\{A(\alpha)\}\right] \theta=L_{j} \cap\{B(\alpha)\}}
\end{aligned}
$$

Proof. (i). To prove the necessity of (4.1) we assume that the extension is already established, that is we assume the existence of $G^{*} \supseteq G$ and an endomorphism $\theta^{*}$ which extends $\mu(\alpha)$ for every $\alpha \in \Sigma$ to $G^{*}$ such that $\theta^{*}$ is an isomorphism on $G^{*}\left(\theta^{*}\right)^{m}$.

For any $g^{*} \in G^{*}, g^{*} \theta^{*}$ is uniquely defined. In particular the map $w\left(a_{\mathrm{t}}\right) \theta^{*}$ of any word $w\left(a_{\mathfrak{\tau}}\right) \in\{A(\alpha)\} \subseteq G^{*}$ is uniquely defined. Since $\theta^{*}$ extends $\mu(\alpha)$ for every $\alpha \in \Sigma$ then

$$
w\left(a_{\tau}\right) \theta^{*}=w\left(a_{\tau} \mu(\tau)\right)=w\left(a_{\tau}\right) \theta
$$

and thus the mapping $\theta$ is one-valued.

Moreover since $\theta^{*}$ extends $\theta$ then for any two words $w\left(a_{\rho}\right), w_{1}\left(a_{\mathfrak{r}}\right) \in\{A(\alpha)\}$ we have

$$
\begin{aligned}
{\left[w\left(a_{\rho}\right) w_{1}\left(a_{\tau}\right)\right] \theta } & =\left[w\left(a_{\rho}\right) w_{1}\left(a_{\mathfrak{t}}\right)\right] \theta^{*} \\
& =\left[w\left(a_{\rho}\right)\right] \theta^{*}\left[w_{1}\left(a_{\tau}\right)\right] \theta^{*} \\
& =\left[w\left(a_{\rho}\right)\right] \theta \cdot\left[w_{1}\left(a_{\tau}\right)\right] \theta
\end{aligned}
$$

which shows that $\theta$ is a homomorphism.

The proof that (4.2) is necessary is the same as in (1).

(ii). To prove the sufficiency of the conditions we put

$$
A_{1}=\{A(\alpha)\}, \quad B_{1}=\{B(\alpha)\} .
$$

Then $\theta$ becomes a partial endomorphism of $G$ which maps $A_{1}$ onto $B_{1}$. Thus because of (4.2) we can extend $\theta$ to a total endomorphism $\theta^{*}$ of $G^{*} \supseteq G$ such that $\theta^{*}$ acts as an isomorphism on $G^{*}\left(\theta^{*}\right)^{m}$. Since $\theta$ extends $\mu(\alpha)$ for every $\alpha \in \Sigma$, then so does $\theta^{*}$.

This completes the proof of Theorem 3. 


\section{REFERENCES}

(1) C. G. Chehata, An embedding theorem for groups, Proc. Glasgow Math. Assoc. 4 (1960), 140-143.

(2) C. G. Chehata, Generalisation of an embedding theorem for groups, Proc. Glasgow Math. Assoc., 4 (1960), 171-177.

(3) B. H. Neumann and Hanna Neumann, Extending partial endomorphisms of groups, Proc. London Math. Soc. (3) 2 (1952), 337-348.

FACULTY OF SCIENCE

THE UNIVERSITY

ALEXANDRIA, EGYPT 